BULL. AUSTRAL. MATH. SOC.

VOL. 30 (1984), 395-410

\title{
MEROMORPHIC STARLIKE UNIVALENT FUNCTIONS
}

\section{V.V. ANH AND P.D. TUAN}

Let $B$ be the class of functions $w(z)$ regular in $|z|<1$ and satisfying $w(0)=0,|w(z)|<1$ in $|z|<1$. We denote by $P(A, B),-1 \leq B<A \leq 1$, the class of functions $p(z)=1+p_{1} z+\ldots$ regular in $|z|<1$ and such that $p(z)=[1+A w(z)] \Lambda 1+B w(z)]$ for some $w(z) \in B$. This paper establishes sharp lower and upper bounds on $|z|=r<1$ for the functional

$$
\operatorname{Re}\left\{\gamma p(z)-\frac{z p^{\prime}(z)}{p(z)}\right\}, \quad \gamma \leq 1
$$

where $p(z)$ varies in $P(A, B)$. The results are then used to study certain geometric properties of the corresponding class of meromorphic starlike univalent functions $\sum^{*}(A, B)=$ $\left\{f(z)=\frac{1}{z}+a_{0}+a_{1} z+\ldots ;-z f^{\prime}(z) / f(z) \in P(A, B),|z|<1\right\}$.

\section{Introduction}

Let $B$ be the class of functions $w(z)$ regular in the unit disc $\Delta=\{z ;|z|<1\}$ and satisfying the conditions $w(0)=0,|\omega(z)|<1$ in $\Delta$. We denote by $P(A, B),-1 \leq B<A \leq 1$, the class of functions

defined by

$$
p(z)=1+p_{1} z+p_{2} z^{2}+\cdots
$$

Received 22 May 1984.

Copyright Clearance Centre, Inc. Serial-fee code: 0004-9727/84 $\$ A 2.00+0.00$. 


$$
p(z)=\frac{1+A w(z)}{1+B w(z)}, \quad z \in \Delta
$$

for some $w(z) \in B$. The definition of this class is a generalisation of the classical result that any regular function $p(z)=1+p_{1} z+p_{2} z^{2}+\cdots$ such that $\operatorname{Re}\{p(z)\}>0$ in $\Delta$ can be written in the form

$$
p(z)=\frac{1+w(z)}{1-w(z)}, \quad w(z) \in B
$$

In [2], Janowski introduced the following general class of starlike univalent functions:

$$
S^{*}(A, B)=\left\{f(z)=z+a_{2} z^{2}+\ldots ; z f^{\prime}(z) / f(z) \in P(A, B), \quad z \in \Delta\right\} .
$$

Given particular values for $A, B, S^{*}(A, B)$ reduces to known subclasses of starlike functions such as (see [2])

$$
\begin{array}{ll}
S_{\alpha}^{*} \equiv S^{*}(1-2 \alpha,-1), & S^{*}(M) \equiv S^{*}(1,1 / M-1), \\
S^{*}(\beta) \equiv S^{*}(\beta,-\beta), & S^{*}(\beta) \equiv S^{*}(\beta, 0) .
\end{array}
$$

In this paper we study the meromorphic counterpart of $S^{*}(A, B)$, namely, the class

$$
\Sigma^{*}(A, B)=\left\{f(z)=\frac{1}{z}+a_{0}+a_{1} z+\ldots ;-z f^{\prime}(z) / f(z) \in P(A, B), z \in \Delta\right\} .
$$

Replacing $A, B$ by appropriate values, we obtain special cases corresponding to those for $S^{*}(A, B)$; in particular, $\Sigma_{\alpha}^{*} \equiv \Sigma^{*}(1-2 \alpha,-1)=\left\{f(z)=1 / z+a_{0}+a_{1} z+\ldots ; \operatorname{Re}\left\{-\frac{z f^{\prime}(z)}{f(z)}\right\}>\alpha, 0 \leq \alpha<1, z \in \Delta\right\}$, $L^{*}[\alpha] \equiv \Sigma^{*}(\alpha,-\alpha)=\left\{f(z)=1 / z+a_{0}+a_{1} z+\ldots ;\left|\left(\frac{z f^{\prime}(z)}{f(z)}+1\right) /\left(\frac{z f^{\prime}(z)}{f(z)}-1\right)\right|<\alpha\right.$, $0<\alpha \leq 1, \quad z \in \Delta\}$, $L^{*}(M) \equiv \Sigma^{*}(1,1 / M-1)=\left\{f(z)=1 / z+a_{0}+a_{1} z+\ldots ;\left|\frac{z f^{\prime}(z)}{f(z)}+M\right|<M, M>\frac{1}{2}, z \in \Delta\right\}$, $L_{(\alpha)}^{*} \equiv L^{*}(\alpha, 0)=\left\{f(z)=1 / z+a_{0}+a_{1} z+\ldots ;\left|\frac{z f^{\prime}(z)}{f(z)}+1\right|<\alpha, 0<\alpha \leq 1, z \in \Delta\right\}$. 
The classes $\sum_{\alpha}^{*}, \Sigma^{*}[\alpha]$ and $\Sigma^{*}(M)$ were investigated by Pommerenke [5], Padmanabhan [4] and Wiatrowski [6] respectively.

Karunakaran [3] recently considered $\sum^{*}(A, B)$ where $A, B$ are restricted by the conditions $-1 \leq B \leq 0, B<A \leq-B$. These conditions are not general enough to cover such cases as $\sum^{*}(M)$ in which $B>0$ for $\frac{1}{2}<M<1$, and $\sum_{\cdot(\alpha)}^{*}$ defined above. This paper deals with $\sum^{*}(A, B)$ where $A, B$ vary in the complete range $-1 \leq B<A \leq 1$.

Problems over $\sum^{*}(A, B)$ such as distortion bounds, radius of convexity may be transformed into the extremal problems

$$
\min _{p(z) \in P(A, B)} \min _{|z|=r<1} \operatorname{Re}\left\{\gamma p(z)-\frac{z p^{\prime}(z)}{p(z)}\right\},
$$

$$
\max _{p(z) \in P(A, B) \quad \max _{|z|=z<1}} \operatorname{Re}\left\{\gamma p(z)-\frac{z p^{\prime}(z)}{p(z)}\right\},
$$

where $\gamma \leq 1$. Problems (1.2) and (1.3), which are of interest in their own right, will be solved in section 2. The results obtained will then be used to derive the radius of convexity for $\sum^{*}(A, B)$ and the distortion bounds for $\sum_{\alpha}^{*}$ and $\Sigma^{*}[\alpha]$.

\section{The extremal problems}

From the definition of $P(A, B)$ we have that

$$
p(z)<\frac{1+A z}{1+B z} \quad, \quad z \in \Delta,
$$

for every $p(z) \in P(A, B)$. Thus, an application of the subordination Principle yields that the image of $|z| \leq r$ under every $p(z) \in P(A, B)$ is contained in the disc

$$
|p(z)-a| \leq d,
$$

where 


$$
a=\frac{1-A B r^{2}}{1-B^{2} r^{2}} \quad, \quad d=\frac{(A-B) r}{1-B^{2} r^{2}}
$$

From (2.1) and (2.2), it follows immediately that if $p(z) \in P(A, B)$, then, on $|z|=r<1$,

$$
\frac{1-A r}{1-B r} \leq \operatorname{Re}\{p(z)\} \leq|p(z)| \leq \frac{1+A r}{1+B r}
$$

The bounds are attained for the function $p(z)=(1+A z) /(1+B z)$.

The basic tool which we rely upon to handle problems (1.2) and (1.3) over $P(A, B)$ is the following inequality known as Dieudonne's Lemma (see Duren [1, p. 25]).

LEMMA 2.1. If $w(z) \in B$, then for $|z|<1$,

$$
\left|z w^{\prime}(z)-w(z)\right| \leq \frac{|z|^{2}-|w(z)|^{2}}{1-|z|^{2}} .
$$

THEOREM 2.2. If $p(z) \in P(A, B),-(1+B) /(A-B) \leq \gamma \leq 1$, then on $|z|=r<1$,

$$
\operatorname{Re}\left\{\gamma p(z)-\frac{z p^{\prime}(z)}{p(z)}\right\} \geq\left\{\begin{array}{l}
\frac{\gamma-[(1-2 \gamma) A-B] r+\gamma A^{2} r^{2}}{(1+A r)(1+B r)}, \\
-\frac{A+B}{A-B}+\frac{2}{(A-B)\left(1-r^{2}\right)}\left[\left(L_{1} K_{1}\right)^{\frac{3}{2}}-\left(1-A B r^{2}\right)\right], R_{2} \geq R_{1},
\end{array},\right.
$$

where $R_{1}=\left(L_{1} / K_{1}\right)^{\frac{1}{2}}, R_{2}=(1+A r) /(1+B r), L_{1}=(1+A)\left(1-A r^{2}\right)$, $K_{1}=\gamma(A-B)\left(1-r^{2}\right)+(1+B)\left(1-B r^{2}\right)$. The result is sharp.

Proof. From the representation (1.1) of $p(z)$ we deduce that

$$
\gamma p(z)-\frac{z p^{\prime}(z)}{p(z)}=\gamma \frac{1+A w(z)}{1+B w(z)}-(A-B) \frac{z w^{\prime}(z)}{[1+A w(z)][1+B w(z)]} .
$$

Applying Dieudonné's Lemma to the second term of the right hand side we find 


$$
\begin{aligned}
\operatorname{Re}\left\{r p(z)-\frac{z p^{\prime}(z)}{p(z)}\right\} \geq & -\frac{A+B}{A-B}+\frac{1}{A-B} \operatorname{Re}\left\{[\gamma(A-B)+B] p(z)+\frac{A}{p(z)}\right\} \\
& -\frac{r^{2}|B p(z)-A|^{2}-|1-p(z)|^{2}}{(A-B)\left(1-r^{2}\right)|p(z)|}
\end{aligned}
$$

Put $p(z)=R e^{i \theta}$, where $R \in[a-d, a+d]$ with $a, d$ given by (2.2) and denote the right-hand side of $(2.5)$ by $S(R, \theta)$, then

$$
\begin{aligned}
S(R, \theta)=-\frac{A+B}{A-B} & +\frac{1}{A-B}\left\{\left[(\gamma(A-B)+B) R+\frac{A}{R}-\frac{2\left(1-A B r^{2}\right)}{1-r^{2}}\right] \cos \theta\right. \\
& \left.+\frac{1-A^{2} r^{2}}{1-r^{2}} \cdot \frac{1}{R}+\frac{1-B^{2} r^{2}}{1-r^{2}} \cdot R\right\}
\end{aligned}
$$

Now,

$$
\frac{\partial S}{\partial \theta}=\frac{\sin \theta}{A-B} T(R)
$$

where

$$
\begin{aligned}
T(R) & =2 \frac{1-A B r^{2}}{1-r^{2}}-\frac{A}{R}-[\gamma(A-B)+B] R \\
& \geq 2 \frac{1-A B r^{2}}{1-r^{2}}-\left(\frac{1}{R}+R\right) \text { as } A \leq 1 \text { and } \gamma \leq 1 .
\end{aligned}
$$

Denote the right-hand side by $F(R)$; then $d F / d R=1 / R^{2}-1$. Since $R \in[a-d, a+d]$ and $a-d<1, a+d>1$, the minimum of $F(R)$ is attained at either $R=a-d$ or $R=a+d$. Now,

$$
\begin{aligned}
E(a-d) & =2 \frac{1-A B r^{2}}{1-r^{2}}-\frac{1-B r}{1-A r}-\frac{1-A r}{1-B r} \\
& =\frac{r^{2}\left[\left(1-A^{2}\right)(1-B r)^{2}+\left(1-B^{2}\right)(1-A r)^{2}\right]}{\left(1-r^{2}\right)(1-A r)(1-B r)}>0 .
\end{aligned}
$$

Also,

$$
F(a+d)=2 \frac{1-A B r^{2}}{1-r^{2}}-\frac{1+B r}{1+A r}-\frac{1+A r}{1+B r}
$$


400

$$
\begin{aligned}
& \text { V. V. Anh and P. D. Tuan } \\
& =\frac{r^{2}\left[\left(1-A^{2}\right)(1+B r)^{2}+\left(1-B^{2}\right)(1+A r)^{2}\right]}{\left(1-r^{2}\right)(1+A r)(1+B r)}>0 .
\end{aligned}
$$

Thus $T(R)>0$. And so, the minimum of $S(R, \theta)$ on the disc $|p(z)-a| \leq d$ is attained when $\theta=0$ and $R \in[a-d, a+d]$. Setting $\theta=0$ in $(2.6)$ we get

$$
S(R, 0)=-\frac{A+B}{A-B}+\frac{1}{A-B}\left\{\left[\gamma(A-B)+B+\frac{1-B^{2} r^{2}}{1-r^{2}}\right] R+\left(A+\frac{1-A^{2} r^{2}}{1-r^{2}}\right) \frac{1}{R}-2 \frac{1-A B r^{2}}{1-r^{2}}\right\}
$$

which yields

$$
\left.\frac{d S(R, 0)}{d R}=\frac{1}{A-B} \gamma(A-B)+B+\frac{1-B^{2} r^{2}}{1-r^{2}}-\frac{(1+A)\left(1-A r^{2}\right)}{1-r^{2}} \cdot \frac{1}{R^{2}}\right]
$$

In the above expression we have that

$$
\gamma(A-B)+B+\frac{1-B^{2} r^{2}}{1-r^{2}} \geq \gamma(A-B)+B+1 \geq 0
$$

if $\gamma \geq-(1+B) /(A-B)$. Thus for $-(1+B) /(A-B) \leq \gamma \leq 1$, the minimum of $S(R, 0)$ occurs at $R=R_{1}$ if $R_{1} \in[a-d, a+d]$, its value being

$$
S\left(R_{1}, 0\right)=-\frac{A+B}{A-B}+\frac{2}{(A-B)\left(1-r^{2}\right)}\left[\left(L_{1} K_{1}\right)^{\frac{3}{2}}-\left(1-A B r^{2}\right)\right]
$$

We next want to show that $R_{1}>a-d$. Indeed, for $\gamma$ in the range $-(1+B) /(A-B) \leq \gamma \leq 1$, we have

$$
\frac{(1+A)\left(1-A r^{2}\right)}{\gamma(A-B)\left(1-r^{2}\right)+(1+B)\left(1-B r^{2}\right)}>\frac{1-A r^{2}}{1-B r^{2}}
$$

if and only if $1-B r^{2}>\gamma\left(1-r^{2}\right)$, that is, if and only if $1>(B-\gamma) r^{2} /(1-\gamma)$, which is always true as $(B-\gamma) /(1-\gamma)<1$ for $\gamma \leq 1$. Consequently,

$$
R_{1}^{2}>\frac{1-A r^{2}}{1-B r^{2}}>\frac{1-A r}{1-B r}>\left(\frac{1-A r}{1-B r}\right)^{2}=(a-d)^{2}
$$


In other words, $R_{1}>a-d$. However, $R_{1}$ is not always less than $a+d$. For $R_{1} \geq a+d=R_{2}$, the minimum of $S(R, 0)$ occurs at $R=R_{2}$, its value being

$$
S\left(R_{2}, 0\right)=\frac{\gamma-[(1-2 \gamma) A-B] r+\gamma A^{2} r^{2}}{(1+A r)(1+B r)}
$$

The result is sharp for the function $p_{1}(z)=(1+A z) /(1+B z)$ for $R_{1} \geq R_{2}$ and the function $P_{2}(z)=\left[1+A w_{2}(z)\right] /\left[1+B w_{2}(z)\right]$ for $R_{2} \geq R_{1}$, where $w_{2}(z)=z\left(z-c_{2}\right) /\left(1-c_{2} z\right)$ with $c_{2}$ being determined from the equation $\operatorname{Re}\left\{\left[1+A w_{2}(z)\right] /\left[1+B w_{2}(z)\right]\right\}=R_{1}$ at $z=r$.

THEOREM 2.3. If $p(z) \in P(A, B), \gamma \leq 1$, then on $|z|=r<1$

$$
\operatorname{Re}\left\{\gamma p(z)-\frac{z p^{\prime}(z)}{p(z)}\right\} \leq\left\{\begin{array}{ll}
\frac{\gamma+[(1-2 \gamma) A-B] r+\gamma A^{2} r^{2}}{(1-A r)(1-B r)}, & R_{3} \leq R_{4}, \\
-\frac{A+B}{A-B}+\frac{2}{(A-B)\left(1-r^{2}\right)}\left\{1-A B r^{2}-\left(L_{2} K_{2}\right)^{\frac{3}{2}}\right], & R_{4} \leq R_{3},
\end{array},\right.
$$

where $R_{3}=\left(L_{2} / K_{2}\right)^{\frac{3}{2}}, R_{4}=(1-A r) /(1-B r), L_{2}=(1-A)\left(1+A r^{2}\right)$, $K_{2}=(1-B)\left(1+B r^{2}\right)-\gamma(A-B)\left(1-r^{2}\right)$. The result is sharp.

Proof. The same argument as in the proof of Theorem 2.2 yields

$$
\begin{aligned}
\operatorname{Re}\left\{\gamma p(z)-\frac{z p^{\prime}(z)}{p(z)}\right\} \leq & -\frac{A+B}{A-B}+\frac{1}{A-B} \operatorname{Re}\left\{[\gamma(A-B)+B] p(z)+\frac{A}{p(z)}\right\} \\
& +\frac{r^{2}|B p(z)-A|^{2}-|1-p(z)|^{2}}{(A-B)\left(1-r^{2}\right)|p(z)|} .
\end{aligned}
$$

Put $p(z)=a+u+i v$ and denote the right-hand side of $(2.7)$ by $S(u, v)$ then

(2.8) $S(u, v)=-\frac{A+B}{A-B}+\frac{1}{A-B}\left[[\gamma(A-B)+B](a+u)+\frac{A(a+u)}{R^{2}}+\frac{1-B^{2} r^{2}}{1-r^{2}} \cdot \frac{d^{2}-u^{2}-v^{2}}{R}\right\}$, 
so that

$$
\frac{\partial S}{\partial v}=-\frac{1}{A-B} \cdot \frac{v}{R^{4}} T(u, v),
$$

where

$$
\begin{aligned}
T(u, v) & =2 A(a+u)+\frac{1-B^{2} r^{2}}{1-r^{2}}\left[2 R^{3}+\left(d^{2}-u^{2}-v^{2}\right) R\right] \\
& \geq 2(a+u)\left[A+\frac{1-B^{2} r^{2}}{1-r^{2}}(a-d)^{2}\right] .
\end{aligned}
$$

Now,

$$
\begin{aligned}
A+\frac{1-B^{2} r^{2}}{1-r^{2}}(a-d)^{2} \geq A+(a-d)^{2} & =\frac{(1+B)(1-A r)^{2}+(A-B)\left(1-A B r^{2}\right)}{(1-B r)^{2}} \\
& >0 .
\end{aligned}
$$

Hence $T(u, v)>0$ and the maximum of $S(u, v)$ on the disc $|p(z)-a| \leq d$ is attained when $v=0$ and $u \in[-d, d]$. Putting $v=0$ in (2.8) gives

$$
S(u, 0)=-\frac{A+B}{A-B}+\frac{1}{A-B}\left\{\left(A-\frac{1-A^{2} r^{2}}{1-r^{2}}\right) \frac{1}{a+u}+\left[\gamma(A-B)+B-\frac{1-B^{2} r^{2}}{1-r^{2}}\right](a+u)+2 \frac{1-A B r^{2}}{1-r^{2}}\right\}
$$

which yields

$$
\frac{d S(u, 0)}{d u}=\frac{1}{(A-B)\left(1-r^{2}\right)}\left[\gamma(A-B)\left(1-r^{2}\right)-(1-B)\left(1+B r^{2}\right)+(1-A)\left(1+A r^{2}\right) \frac{1}{(a+u)^{2}}\right] \text {. }
$$

Now $(1-B)\left(1+B r^{2}\right)-\gamma(A-B)\left(1-r^{2}\right)>0$ if and only if

$$
\gamma<\frac{1-B}{A-B} \cdot \frac{1+B r^{2}}{1-r^{2}}
$$

Since $1-B \geq A-B$ and $\left(1+B r^{2}\right) /\left(1-r^{2}\right) \geq 1$, the restriction $\gamma \leq 1$ shows that the above condition is satisfied. Hence with $\gamma \leq 1$, we see that $d S(u, 0) / d u$ vanishes at $u_{0}=\left(L_{2} / K_{2}\right)^{\frac{1}{2}}-a$. Thus the maximum of $S(u, 0)$ occurs at $u=u_{0}$ if $u_{0} \in[-d, d]$, its value being

$$
S\left(u_{0}, 0\right)=-\frac{A+B}{A-B}+\frac{2}{(A-B)\left(1-r^{2}\right)}\left[1-A B r^{2}-\left(L{ }_{2} K_{2}\right)^{\frac{1}{2}}\right] \text {. }
$$




\section{Meromorphic starlike univalent functions}

Now, an easy calculation shows that

$$
\frac{(1-A)\left(1+A r^{2}\right)}{(1-B)\left(1+B r^{2}\right)-\gamma(A-B)\left(1-r^{2}\right)}<\frac{1+A r^{2}}{1+B r^{2}}
$$

if and only if $\gamma\left(1-r^{2}\right)<1+B r^{2}$, which holds for $\gamma \leq 1$. Hence

$$
\left(a+u_{0}\right)^{2}<\frac{1+A r^{2}}{1+B r^{2}}<(a+d)^{2},
$$

that is, $u_{0}<d$. However, it is not necessary that $u_{0}>-d$. For $u_{0} \leq-d$, that is, $R_{3} \leq R_{4}$, the maximum of $s(u, 0)$ occurs at $u_{0}=-d$, its value being

$$
S(-d, 0)=\frac{\gamma+[(1-2 \gamma) A-B] r+\gamma A^{2} r^{2}}{(1-A r)(1-B r)}
$$

The result is sharp for the function $p_{1}(z)=(1+A z) /(1+B z)$ for $R_{3} \leq R_{4}$ and the function $\left.p_{3}(z)=\left[1+A w_{3}(z)\right] \Lambda 1+B w_{3}(z)\right]$ for $R_{4} \leq R_{3}$. where $w_{3}(z)=z\left(z-c_{3}\right) /\left(1-c_{3} z\right)$ with $c_{3}$ such that $\left.\operatorname{Re}\left\{\left[1+A w_{3}(z)\right] \Lambda 1+B w_{3}(z)\right]\right\}=R_{3}$ at $z=-r$.

\section{The class $\sum^{*}(A, B)$}

This section establishes the radius of convexity and the bounds for $|f(z)|$ for $\sum^{*}(A, B)$. The bounds for $\left|f^{\prime}(z)\right|$ over $\sum^{*}(A, B)$ are not known. However, we shall determine these bounds for two special cases of $\Sigma^{*}(A, B)$, namely, $\sum_{\alpha}^{*}$ and $\Sigma^{*}[\alpha]$.

THEOREM 3.1. The radius of convexity of $\left[^{*}(A, B)\right.$ is given by the smallest root in $(0,1]$ of

(i) $A^{2} r^{2}+(A+B) r+1=0$, for $R_{2} \leq R_{1}$,

(ii) $\left(4 A^{2}+3 A+B\right) r^{4}-2\left[2(1+A)^{2}+A-B\right] r^{2}+4+3 A+B=0$, for $R_{1} \leq R_{2}$, $R_{1}, R_{2}$ being as given in Theorem 2.2 with $\gamma=1$. 
Proof. For $f(z) \in \sum^{*}(A, B)$, we deduce

$$
-\left[1+\frac{z f^{\prime \prime}(z)}{f^{\prime}(z)}\right]=p(z)-\frac{z p^{\prime}(z)}{p(z)}, \quad p(z) \in P(A, B) .
$$

The result now follows from Theorem 2.2 with $\gamma=1$ and is sharp for the functions $f_{1}(z)$ for $R_{2} \leq R_{1}$ and $f_{2}(z)$ for $R_{1} \leq R_{2}$, where $f_{1}(z)$, $f_{2}(z)$ are given by

$$
-\frac{z f_{i}^{\prime}(z)}{f_{i}(z)}=p_{i}(z) \quad, \quad i=1,2,
$$

$p_{1}(z), p_{2}(z)$ being extremal for Theorem 2.2 .

As a special case of Theorem 3.1 , we determine the radius of convexity of the class $\sum_{(\alpha)}^{*}$ of functions $f(z)=1 / z+a_{0}+a_{1} z+\ldots$ for which

$$
\left|\frac{z f^{\prime}(z)}{f(z)}+1\right|<\alpha, 0<\alpha \leq 1, z \in \Delta
$$

COROLLARY 3.2. The radius of convexity of $\sum_{(\alpha)}^{*}$ is

$$
\sigma=\left\{\left[2+5 \alpha+2 \alpha^{2}-2(1+\alpha)\left(1+\alpha^{2}\right)^{\frac{1}{2}}\right] / \alpha(4 \alpha+3)\right\}^{\frac{1}{2}} \text {. }
$$

Proof. For $f(z) \in \sum_{(\alpha)}^{*}$ we may write

$$
-\frac{z f^{\prime}(z)}{f(z)}=p(z), \quad z \in \Delta
$$

where $p(z)$ satisfies the condition

$$
|p(z)-1|<\alpha, 0<\alpha \leq 1, z \in \Delta .
$$

Put $w(z)=[p(z)-1] / \alpha ;$ then $w(z) \in B$ and $p(z)=1+\alpha w(z)$. Hence $p(z) \in P(\alpha, 0)$. Theorem 3.1 with $A=\alpha, B=0$ gives, for $R_{1} \leq R_{2}$, the radius of convexity of $f(z)$ to be the smallest root in $(0,1]$ of the equation

$$
\alpha(4 \alpha+3) r^{4}-2\left(2+5 \alpha+2 \alpha^{2}\right) r^{2}+4+3 \alpha=0 .
$$

It is clear that the only root in $(0,1)$ of this equation is $\sigma$. Now, the condition $R_{2} \leq R_{1}$ with $A=\alpha, B=0, \gamma=1$ is equivalent to

$$
-2(1+\alpha)-\alpha(2+\alpha) r+2 \alpha r^{2}+\alpha^{2} r^{3} \leq 0 \text {, }
$$




\section{Meromorphic starlike univalent functions}

which always holds for $0<\alpha \leq 1,0<r<1$. Hence the case $R_{2} \leq R_{1}$ does not exist for the class $\Sigma_{(\alpha)}^{*}$. The proof is therefore completed.

To obtain bounds for $|f(z)|$ over $\sum^{*}(A, B)$, we observe that $f(z) \in L^{*}(A, B)$ if and only if $1 / f(z) \in S^{*}(A, B)$. Hence an application of Theorem 4 of [2] gives

COROLLARY 3.3. Let $f(z) \in \sum^{*}(A, B)$; then on $|z|=r<1$,

$$
\begin{gathered}
r^{-1}(1+B r)^{(B-A) / B} \leq|f(z)| \leq r^{-1}(1-B r)^{(B-A) / B}, \text { if } B \neq 0, \\
r^{-1} \exp (-A r) \leq|f(z)| \leq r^{-1} \exp (A r), \text { if } B=0 .
\end{gathered}
$$

The function $f(z)$ defined by

$$
-\frac{z f^{\prime}(z)}{f(z)}=\frac{1+A z}{1+B z}, \quad z \in \Delta
$$

shows that the bounds are sharp.

We next derive bounds for $\left|f^{\prime}(z)\right|$ for two subclasses of $\sum^{*}(A, B)$ namely, $\sum_{\alpha}^{*} \equiv \Sigma^{*}(1-2 \alpha,-1)$ and $\sum^{*}[\alpha] \equiv \Sigma^{*}(\alpha,-\alpha)$.

THEOREM 3.4 Let $f(z) \in \sum_{\alpha}^{*}, \beta=1-2 \alpha ;$ then on $|z|=r<1$

$$
\left|f^{\prime}(z)\right| \leq\left\{\begin{array}{l}
r^{-2}\left(1-r^{2}\right)^{-1}, \quad \alpha=0, \\
\frac{1}{4 r^{2}}\left[1+\left(\frac{1-\beta r^{2}}{1-r^{2}}\right)^{\frac{2}{2}}\right]^{2}\left\{\frac{(1+\sqrt{\beta})\left[\left(1-\beta r^{2}\right)^{\frac{3}{2}}-\sqrt{\beta}\left(1-r^{2}\right)^{\frac{3}{2}}\right]}{(1-\sqrt{\beta})\left[\left(1-\beta r^{2}\right)^{\frac{3}{2}}+\sqrt{\beta}\left(1-r^{2}\right)^{\frac{1}{2}}\right]}\right\}^{\sqrt{\beta}}, 0<\alpha \leq \frac{3}{2}, \\
\frac{1}{r^{2}}\left[1+\left(\frac{1-\beta r^{2}}{1-r^{2}}\right)^{\frac{1}{2}}\right]^{2} \exp \left\{2 \sqrt{-\beta} \tan ^{-1} \frac{\sqrt{-\beta}\left(1-\left(1-r^{2}\right)^{\frac{3}{2}}\left(1-\beta r^{2}\right)^{\frac{1}{2}}\right]}{1+\beta-\beta r^{2}}\right\},, \frac{1}{2} \leq \alpha \leq \alpha_{0},
\end{array}\right.
$$

where $4 / 5 \leq \alpha_{0}<1$ : 


$$
\left|f^{\prime}(z)\right| \geq\left\{\begin{array}{l}
1 / r^{2}-1, \alpha=0, \\
r^{-2}\left(1-r^{2}, \beta /(1-\alpha)\left\{\frac{1+\left[\left(1+\beta r^{2}\right) /\left(1-r^{2}\right)\right]^{\frac{1}{2}}}{2}\right\}^{-2 \alpha /(1-\alpha)} \times\right. \\
\exp \left\{\frac{2 \alpha \sqrt{\beta}}{1-\alpha} \tan ^{-1} \frac{\sqrt{\beta}\left[1-\left(1-r^{2}\right)^{\frac{2}{2}}\left(1+\beta r^{2}\right)^{\frac{1}{2}}\right]}{2 \alpha+\beta r^{2}}\right\}, 0<\alpha \leq \frac{1}{2}, \\
r^{-2}\left(1-r^{2}\right)^{\beta /(1-\alpha)}\left\{\frac{1+\left[\left(1+\beta r^{2}\right) /\left(1-r^{2}\right)\right]^{\frac{1}{2}}}{2}\right\}^{-2 \alpha /(1-\alpha)} \times \\
\left\{\frac{(1-\sqrt{-\beta})\left[\left(1+\beta r^{2}\right)^{\frac{1}{2}}+\sqrt{-\beta}\left(1-r^{2}\right)^{\frac{1}{2}}\right]}{(1+\sqrt{-\beta})\left[\left(1+\beta r^{2}\right)^{\frac{1}{2}}-\sqrt{-\beta}\left(1-r^{2}\right)^{\frac{1}{2}}\right]} \alpha \sqrt{-\beta} /(1-\alpha), \frac{1}{2} \leq \alpha<1 .\right.
\end{array} .\right.
$$

The results are sharp.

Proof. From the expression

$$
\log \left(z^{2} f^{\prime}(z)\right)=\log \left|z^{2} f^{\prime}(z)\right|+\operatorname{iarg}\left\{z^{2} f^{\prime}(z)\right\},
$$

we derive

$$
2+\operatorname{Re}\left\{\frac{z f^{\prime \prime}(z)}{f^{\prime}(z)}\right\}=r \frac{\partial}{\partial r} \log \left|z^{2} f^{\prime}(z)\right| .
$$

This together with (3.1) give, for $f(z) \in \Sigma_{\alpha}^{*}$,

$$
p \frac{\partial}{\partial r} \log \left|z^{2} f^{\prime}(z)\right|=1-\operatorname{Re}\left\{p(z)-\frac{z p^{\prime}(z)}{p(z)}\right\}, p(z) \in P_{\alpha} .
$$

The condition $R_{1} \leq R_{2}$ of Theorem 2.2 with $A=1-2 \alpha, B=-1, \gamma=1$ is equivalent to

$$
F(\alpha) \equiv-2 r(1+r) \alpha^{2}+\left(r^{2}+5 r+2\right) \alpha-2(1+r) \leq 0 .
$$

Now, $F(0)=-2(1+r)<0, F(1)=r(1-r)>0, F(4 / 5)=-2\left(6 r^{2}-9 r+5\right) / 25<0$

for $0<r<1$. Hence $F(\alpha)$ has a zero in $[4 / 5,1)$. It may be checked that this is the only zero, denoted by $\alpha_{0}$, less than 1 of $F(\alpha)$. Thus for $\alpha \leq \alpha_{0}$, we have $F(\alpha) \leq 0$ for $0<r<1$. And so the case $R_{2} \leq R_{1}$ does not exist for $0 \leq \alpha \leq \alpha_{0}$ when we consider the class $\sum_{\alpha}^{*}$. Theorem 2.2 with $A=1-2 \alpha, B=-1, \gamma=1$ applied to (3.2) yields 


$$
\frac{\partial}{\partial r} \log \left|z^{2} f^{\prime}(z)\right| \leq 2\left\{\frac{1-\left[\left(1-B r^{2}\right)\left(1-r^{2}\right)\right]^{\frac{3}{2}}}{1-r^{2}}\right\}
$$

Hence

$$
\begin{aligned}
\log \left|z^{2} f^{\prime}(z)\right| & \leq 2 \int_{0}^{r} \frac{1-\left[\left(1-\beta t^{2}\right)\left(1-t^{2}\right)\right]^{\frac{2}{2}}}{t\left(1-t^{2}\right)} d t \\
& =2 \int_{0}^{2} \frac{t\left[2(1-\alpha)-\beta t^{2}\right] d t}{\left(1-t^{2}\right)\left\{1+\left(1-t^{2}\right)\left[\left(1-\beta t^{2}\right) /\left(1-t^{2}\right)\right]^{\frac{1}{2}}\right\}} .
\end{aligned}
$$

With the substitution $u=\left[\left(1-B t^{2}\right) /\left(1-t^{2}\right)\right]^{\frac{1}{2}}$, the integration may be carried out to give the upper bound for $\left|f^{\prime}(z)\right|$. To obtain the lower bound for $\left|f^{\prime}(z)\right|$, we note first of all that the condition $R_{4} \leq R_{3}$ of Theorem 2.3 with $A=1-2 \alpha, B=-1, \gamma=1$ is equivalent to the inequality

$$
2+(1+2 \alpha) r+(1-2 \alpha) r^{3} \geq 0 \text {, }
$$

which always holds for $0<r<1,0 \leq \alpha<1$. Hence there is only one case, $R_{4} \leq R_{3}$, for the upper bound of $\operatorname{Re}\left\{p(z)-z p^{\prime}(z) / p(z)\right\}$ with $p(z) \in P_{\alpha}$. This result applied to (3.2) gives

$$
r \frac{\partial}{\partial r} \log \left|z^{2} f^{\prime}(z)\right| \geq \frac{\beta}{1-\alpha}-\frac{1}{(1-\alpha)\left(1-r^{2}\right)}\left\{1+\beta r^{2}-2 \alpha\left[\left(1+\beta r^{2}\right)\left(1-r^{2}\right)\right]^{\frac{1}{2}}\right\} .
$$

Hence

$$
\begin{aligned}
\log \left|z^{2} f^{\prime}(z)\right| \geq \frac{1}{1-\alpha} \int_{0}^{r}\left\{\frac{-2 \beta t}{1-t^{2}}-2 \alpha \frac{1-\left[\left(1+\beta t^{2}\right)\left(1-t^{2}\right)\right]^{\frac{3}{2}}}{t\left(1-t^{2}\right)}\right\} d t \\
\quad=\frac{\beta}{1-\alpha} \log \left(1-r^{2}\right)-\frac{2 \alpha}{1-\alpha} \int_{0}^{r} \frac{t\left(2 \alpha+\beta t^{2}\right) d t}{\left(1-t^{2}\right)\left\{1+\left(1-t^{2}\right)\left[\left(1+B t^{2}\right) /\left(1-t^{2}\right)\right]^{\frac{1}{2}}\right\}} .
\end{aligned}
$$

It follows at once from (3.4) that, for $\alpha=0,\left|f^{\prime}(z)\right| \geq 1 / r^{2}-1$. For $0<\alpha<1$, with the substitution $u=\left[\left(1+\beta t^{2}\right) /\left(1-t^{2}\right)\right]^{\frac{1}{2}}$ and carrying out the integration, we get the lower bound for $\left|f^{\prime}(z)\right|$.

The upper bound for $\left|f^{\prime}(z)\right|$ is attained for the function $f(z)$ defined by 
408

V. V. Anh and P. D. Tuan

$$
-\frac{z f^{\prime}(z)}{f(z)}=p_{2}(z)
$$

while the lower bound for $\left|f^{\prime}(z)\right|$ occurs for the function $f(z)$ defined by

$$
-\frac{z f^{\prime}(z)}{f(z)}=p_{3}(z)
$$

where $p_{2}(z), p_{3}(z)$ are extremal for Theorems 2.2 and 2.3 respectively. Padmanabhan [4] in his work on $S^{*}[\alpha]$ and $S^{*}[\alpha]$ derived the radius of convexity of $\sum^{*}[\alpha]$, while the distortion theorem for this class was not given. Here we prove

THEOREM 3.5. If $f(z) \in \sum^{*}[\alpha]$, then on $|z|=r<1$

$$
\frac{\left(1-r^{2}\right)^{\alpha}}{r^{2}} \leq\left|f^{\prime}(z)\right| \leq \frac{1}{r^{2}\left(1-r^{2}\right)^{\alpha}} \text {. }
$$

The results are sharp.

Proof. Denote by $P[\alpha]$ the class of functions $p(z)=1+p_{1} z+\ldots$ which satisfy the condition

$$
\left|\frac{p(z)-1}{p(z)+1}\right|<\alpha, 0<\alpha \leq 1, z \in \Delta,
$$

that is, $P[\alpha] \equiv P(\alpha,-\alpha)$. For $f(z) \in L^{*}[\alpha]$, we may write

$$
r \frac{\partial}{\partial x} \log \left|z^{2} f^{\prime}(z)\right|=1-\operatorname{Re}\left\{p(z)-\frac{z p^{\prime}(z)}{p(z)}\right\}
$$

as in the proof of Theorem 3.4, where now $p(z) \in P[\alpha]$. The condition $R_{1} \geq R_{2}$ of Theorem 2.2 with $A=\alpha, B=-\alpha, \gamma=1$ is equivalent to $-2(1+\alpha)\left(1-\alpha r^{2}\right) \leq 0$, which is always true for $0<r<1,0<\alpha \leq 1$. Hence the case $R_{2} \leq R_{1}$ does not exist for $p(z) \in P[\alpha]$. Consequently, an application of Theorem 2.2 to (3.5) yields

$$
r \frac{\partial}{\partial r} \log \left|z^{2} f^{\prime}(z)\right| \leq \frac{2 \alpha r^{2}}{1-r^{2}} .
$$

And so, 


$$
\log \left|z^{2} f^{\prime}(z)\right| \leq \int_{0}^{r} \frac{2 \alpha t d t}{1-t^{2}}=-\alpha \log \left(1-r^{2}\right),
$$

that is, $\left|f^{\prime}(z)\right| \leq r^{-2}\left(1-r^{2}\right)^{-\alpha}$. Similarly, we can show that the case $R_{3} \leq R_{4}$ of Theorem 2.3 does not exist for $p(z) \in P[\alpha]$ and the lower bound for $\left|f^{\prime}(z)\right|$ can be derived from Theorem 2.3 with $A=\alpha, B=-\alpha$, $\gamma=1$ and (3.5).

The upper bound for $f^{\prime}(z)$ is attained for the function $f(z)$ defined by

$$
-\frac{z f^{\prime}(z)}{f(z)}=p_{2}(z)
$$

while its lower bound is attained for the function $f(z)$ defined by

$$
-\frac{z f^{\prime}(z)}{f(z)}=p_{3}(z)
$$

$p_{2}(z), p_{3}(z)$ being extremal for Theorems 2.2 and 2.3 respectively.

\section{References}

[1] P. Duren, "Subordination" (Lecture Notes in Mathematics, Springerverlag, 599, 1977).

[2] W. Janowski, "Some extremal problems for certain families of analytic functions I", Ann. Polon. Math., 28 (1973), 298-326.

[3] V. Karunakaran, "On a class of meromorphic starlike functions in the unit disc", Math. Chronicle, 4 (1976), 112-121.

[4] K. S. Padmanabhan, "On certain classes of starlike functions in the unit disk", J. Indian Math. Soc., 32 (1968), 89-103.

[5] c. Pommerenke, "On meromorphic starlike functions", Pacific J. Math., 13 (1963), 221-235. 
410

$$
\text { V. V. Anh and P. D. Tuan }
$$

[6] P. Wiatrowski, "On the radius of convexity of some family of functions regular in the ring $0<|z|<1^{n}$, Ann. Polon. Math., 25 (1971), 85-98.

Department of Mathematics, Queensland Institute of Technology,

P. O. BOX 2434,

BRISBANE， 4001

QUEENSLAND,

AUSTRALIA.

First Interstate Bank of California,

600 South Spring street,

LOS ANGELES, CA90014,

U.S.A. 